\title{
Gamma-ray bursts in normal and extreme star-forming galaxies
}

\author{
Neil Trentham, ${ }^{1 \star}$ Enrico Ramirez-Ruiz ${ }^{1}$ and A. W. Blain ${ }^{1,2}$ \\ ${ }^{1}$ Institute of Astronomy, University of Cambridge, Madingley Road, Cambridge CB3 OHA \\ ${ }^{2}$ Astronomy Department, Caltech 105-24, Pasadena, CA 91125, USA
}

Accepted 2002 April 18. Received 2002 April 4; in original form 2001 September 14

\begin{abstract}
We discuss how gamma-ray burst (GRB) optical afterglows and multiwavelength observations of their host galaxies can be used to obtain information about the relative amounts of star formation happening in optical and submillimetre galaxies. That such an analysis will be possible follows from the currently favoured idea that GRBs are closely linked with highmass star formation. Studying GRB host galaxies offers a method of finding low-luminosity submillimetre galaxies, which cannot be identified either in optical Lyman break surveys, because so much of their star formation is hidden by dust, or in submillimetre surveys, because their submillimetre fluxes are close to or below the confusion limit. Much of the star formation in the Universe could have occurred in such objects, so searching for them is an important exercise. From current observations, GRB host galaxies appear to be neither optically luminous Class-2 SCUBA galaxies like SMM J02399 - 0136 or SMM J14011 + 0252, nor galaxies containing dense molecular cores like local ultraluminous infrared galaxies (ULIGs), but rather some intermediate kind of galaxy. The host galaxy of GRB 980703 is a prototype of this kind of galaxy.
\end{abstract}

Key words: cosmology: observations - gamma-rays: bursts - infrared: galaxies.

\section{INTRODUCTION}

Much of the star-formation activity happening in the Universe at redshifts $z>1$ is hidden by dust (e.g. Blain et al. 1999a,b, 2002; Chary \& Elbaz 2001). At low redshift $(z \ll 1)$, the amount of star formation in optical/ultraviolet (UV) galaxies accounts for almost all of the star formation happening in the Universe, but at higher redshift it could well be much lower, due to the existence of many heavily dust-enshrouded submillimetre galaxies.

The populations of galaxies that are bright at optical/UV and submillimetre wavelengths are largely disjoint. The optical/UV galaxies which dominate at high- $z$ are Lyman-break galaxies (Steidel et al. 1999) with typical 850- $\mu \mathrm{m}$ submillimetre fluxes of about $0.1 \mathrm{mJy}$ (Peacock et al. 2000). The submillimetre galaxies which are most common at high $z$ tend to have optical magnitudes $I>26$ (Smail et al. 2002). The reason that these galaxies are so faint is presumably due to internal dust extinction removing most of the emitted restframe ultraviolet light, which corresponds to observed-frame optical light. This internal extinction is similarly important in the most luminous dust-enshrouded galaxies locally, the ULIGs (Sanders \& Mirabel 1996), which are perhaps the most plausible local counterparts to the high- $z$ submillimetre galaxies, but tend to have very low ultraviolet fluxes (Goldader et al. 2002).

^E-mail: trentham@ast.cam.ac.uk
Despite what is written in the previous paragraph, a few of the best-studied high- $z$ submillimetre galaxies have properties quite unilke faint field galaxies with $I>26$. These include

(i) Class-2 SCUBA sources (Ivison et al. 2000; Smail et al. 2002) like SMM J02399 - 0136 (Ivison et al. 1998) and SMM J14011+ 0252 (Ivison et al. 2001), which are extremely luminous at optical wavelengths and have rest-frame intrinsic $B$ magnitudes more than one magnitude brighter than any of the luminous Lyman-break galaxies studied by Pettini et al. (2001). The star-formation rates of Class 2 SCUBA sources implied by both rest-frame $B$-band luminosities and $\mathrm{H} \alpha$ luminosities are high, comparable to those derived from the submillimetre luminosities. The star formation in these sources could be only lightly obscured, leading to the bright optical fluxes;

(ii) Extremely red objects (EROs), or Class-1 SCUBA sources (Smail et al. 2002). These could be more obscured versions of the Class 2s, in which most rest-frame optical light escapes but not most rest-frame ultraviolet light.

These two types of object are easy to study in detail because optical or near-infrared identifications are readily available, but most evidence (Smail et al. 2002) suggests that they do not represent the bulk of the submillimetre galaxy population. Indeed, the difficulty in obtaining unambiguous optical identifications is one of the most important hindrances to our understanding the submillimetre population. 
A current observational problem is that only the brightest submillimetre galaxies can be found in direct surveys (using the SCUBA camera on the James Clerk Maxwell Telescope; Holland et al. 1999) owing to confusion at $850-\mu \mathrm{m}$ fluxes below $\approx 2 \mathrm{mJy}$ (Blain, Ivison \& Smail 1998). Yet much of the star formation in the Universe could be happening in submillimetre galaxies with fainter fluxes, given the constraints jointly imposed by the submillimetre and far-infrared backgrounds and source counts (Blain et al. 1999a,b). These objects cannot be found easily in optical field surveys (e.g. Steidel et al. 1999) either, since their optical fluxes are low (Ivison et al. 2000; Smail et al. 2002). This situation may change at low redshifts $(z \leqslant 2)$ in the near future however, with the advent of deep mid- and far-infrared field surveys from the SIRTF satellite (e.g. Lonsdale 2001).

In a separate development, evidence is now accumulating that gamma-ray bursts (GRBs) are intimately linked with the deaths of young massive stars. This is because (i) a natural mechanism for producing a long-duration ( $>2 \mathrm{~s}$ ) burst is the cataclysmic collapse of a massive star leading to a hypernova-type explosion (Woosley 1993; Paczyński 1998; MacFadyen \& Woosley 1999; MacFadyen, Woosley \& Heger 2001); (ii) the GRB host galaxies which have been identified often show signs of ongoing star formation, such as very blue colours and prominent emission lines (Bloom et al. 1998a; Kulkarni et al. 1998), high radio continuum fluxes (Berger, Kulkarni \& Frail 2001), and high submillimetre fluxes (Frail et al. 2002); (iii) iron line and edge features have been identified in two bursts (Piro et al. 2000; Amati et al. 2000), suggesting a link with supernovae. The mass of iron implied by these measurements is uncertain but consistent with the masses of iron produced in corecollapse supernovae; (iv) in at least four bursts a red component with a flux consistent with that of a supernova was discovered several weeks after the burst (Bloom et al. 1999a; Reichart 1999; Björnsson et al. 2001; Lazzati, Covino \& Ghisellini 2002). GRBs are therefore thought to be found in regions of ongoing star formation, since young massive stars do not live long enough to move significantly from the sites of their birth.

An afterglow at rest-frame optical wavelengths has been observed following many GRBs, thought to be caused by the interaction between the relativistic blast wave originating from the central engine and the local interstellar medium (Rees \& Mészáros 1992, 1994; Paczyński \& Xu 1994; Katz 1994; Sari \& Piran 1995; Mészáros \& Rees 1997). Whether or not this optical afterglow reaches the observer is a function of the line-of-sight extinction towards the GRB (Waxman \& Draine 2000; Venemans \& Blain 2001; Draine \& Hao 2001; Fruchter, Krolik \& Rhoads 2001b; Reichart \& Price 2002), which is likely to be very different for bursts happening within submillimetre and optical/UV galaxies.

The line-of-sight extinction to a GRB is, however, complicated by local physical processes. For example, optical/UV/X-ray radiation from the GRB may heat and sublime nearby dust (Waxman \& Draine 2000; Galama \& Wijers 2001; Venemans \& Blain 2001; Fruchter et al. 2001b). However, radiation from the GRB is unlikely to destroy the dust responsible for the bulk of the extinction in submillimetre galaxies since the X-ray/UV flux scales with distance $d$ as $\sim d^{-2}$ so that dust grains at $\gg 10 \mathrm{pc}$ from the GRB are unaffected, and it is dust on these large scales that is responsible for most of the internal extinction. On the other hand, Fruchter et al. (2001b) recently suggested that the dust grains most responsible for internal extinction may indeed be destroyed by strongly beamed X-ray radiation if the electrostatic stresses generated by the X-rays exceed the grain tensile strengths.
If most submillimetre galaxies are optically faint owing to huge amounts of internal extinction, then we expect the optical afterglow of most GRBs occurring within them to be completely extinguished. This would certainly happen were most submillimetre galaxies to be similar to local ULIGs like Arp 220 since there are typically tens of magnitudes of visual extinction to their cores (e.g. see Scoville et al. 1998). Indeed, Ramirez-Ruiz, Trentham \& Blain (2001) suggest this as the reason for undetected optical afterglows in about half the GRBs studied by Lazzati et al. (2002).

Here we assess observations of optical afterglows in conjunction with submillimetre limits to the flux densities of GRB host galaxies. Of particular interest is the possibility of using GRB host galaxies to find low-luminosity submillimetre galaxies which cannot be found in either SCUBA or optical surveys. Interpretations based on current data and prospects for the future are presented. Throughout the paper we assume $H_{0}=65 \mathrm{~km} \mathrm{~s}^{-1} \mathrm{Mpc}^{-1}, \Omega_{\mathrm{m}}=0.3$, and $\Omega_{\Lambda}=0.7$.

\section{OBSERVATIONS OF GRBS IN SUBMILLIMETRE GALAXIES}

\subsection{Submillimetre observations and the host of GRB 010222}

Most current evidence suggests that the fraction of cosmic star formation happening in submillimetre galaxies $f_{\text {submm }}$ is high, with estimates ranging up to about 0.9 . The value of $f_{\text {submm }}$ might however be poorly constrained by current observations owing to uncertainty in the form of both evolution of the luminosity function of infrared galaxies and their spectral energy distributions (SEDs) - compare the results of Blain et al. (1999a,b), Trentham, Blain \& Goldader (1999a), Eales et al. (1999, 2000) and Gispert, Lagache \& Puget (2000). One reason that these functions are relatively poorly constrained is that no redshifts are currently known for high-redshift submillimetre galaxies, except for a small number of Class-2 SCUBA galaxies (Smail et al. 2002), which do not comprise the bulk of the submillimetre galaxy population, and for the host galaxies of GRB 010222 and GRB 000418 (see below). Another reason is that the dust temperatures of the submillimetre galaxies are unknown (they have only been measured for a small number of Class-2 SCUBA galaxies), and the bolometric luminosity of a submillimetre galaxy is a strong function of the dust temperature. Other uncertainties, like the conversion from infrared luminosities to star-formation rates, reflecting uncertainties in the initial stellar mass function, are potentially important too.

Noting the above, in this work we follow Ramirez-Ruiz et al. (2001) and estimate $f_{\text {submm }}=0.82 \pm 0.10$ (based on the models of Blain et al. 1999a,b). Within the submillimetre galaxy population, a fraction $0.10-0.20$ of the total star formation happens in sources with observed $850-\mu \mathrm{m}$ fluxes $S_{850}>4 \mathrm{mJy}$ (see fig. 7 of Ramirez-Ruiz et al. 2001). This transition value of $4 \mathrm{mJy}$ is chosen because it represents an attainable flux threshold for detecting GRB host galaxies, given several hours of observing with SCUBA. Star formation integrated over cosmic time is then partitioned between the various type of galaxies as follows: SCUBA galaxies with $S_{850}>4$ mJy: 0.07-0.18; SCUBA galaxies with $S_{850}<4$ mJy: $0.58-$ 0.83; UV galaxies: $0.08-0.28$. If GRBs are associated with recent star formation, as outlined in Section 1, then they should happen in the three different types of galaxies in these proportions since they can be seen at all the redshifts.

The most complete survey for submillimetre host galaxies of GRBs with afterglows using SCUBA is that of Smith et al. (1999, 2001). Their results are presented in Table 1. In a separate study, 
Table 1. SCUBA measurements for the hosts of GRBs with optical afterglows. The first set of entries are the $3 \sigma$ limits from Smith et al. $(1999,2001)$. The second entries are the measured $850-\mu \mathrm{m}$ flux of the hosts of GRB 010222 and GRB 000418 from Frail et al. (2002) and Berger et al. (2002). The third entry is the $850-\mu \mathrm{m}$ flux inferred for the host of GRB 980703 from the radio measurements of Berger et al. (2001).

\begin{tabular}{lcc}
\hline GRB & $z$ & $850-\mu \mathrm{m} \mathrm{flux}$ \\
\hline 970508 & 0.84 & $<30 \mathrm{mJy}$ \\
971214 & 3.42 & $<3.04 \mathrm{mJy}$ \\
980326 & Unknown & $<8.1 \mathrm{mJy}$ \\
980329 & $<3.9$ & $<3.5 \mathrm{mJy}$ \\
980519 & Unknown & $<7.3 \mathrm{mJy}$ \\
980703 & 0.97 & $<5.4 \mathrm{mJy}$ \\
981220 & Unknown & $<4.9 \mathrm{mJy}$ \\
981226 & Unknown & $<12 \mathrm{mJy}$ \\
991208 & 0.71 & $<4.9 \mathrm{mJy}$ \\
991216 & 1.0 & $<3.5 \mathrm{mJy}$ \\
$000301 \mathrm{C}$ & 2.03 & $<3.6 \mathrm{mJy}$ \\
000630 & Unknown & $<6 \mathrm{mJy}$ \\
000911 & 1.06 & $<2.6 \mathrm{mJy}$ \\
000926 & 2.04 & $<20 \mathrm{mJy}$ \\
010222 & 1.48 & $3.7 \pm 0.53 \mathrm{mJy}$ \\
000418 & 1.18 & $3.6 \pm 0.8 \mathrm{mJy}$ \\
980703 & 0.97 & $1.9 \mathrm{mJy}$ \\
\hline
\end{tabular}

GRB $010222(z=1.476)$ was found to have a time-independent 850 - $\mu \mathrm{m}$ SCUBA detection of $\sim 4 \mathrm{mJy}$, which is likely to originate from the host galaxy (Frail et al. 2002). Interestingly, this burst had a bright optical afterglow, meaning that it did not happen in a region of very heavy obscuration. Yet this is not a Class-2 SCUBA galaxy since its optical afterglow light curve (Holland et al. 2001a) does not flatten at $R \sim 22$. Recently, Berger et al. (2002) detected submillimetre and radio emission consistent with the host galaxy of GRB 000418.

Only five bursts in the Smith et al. $(1999,2001)$ sample have $3 \sigma$ flux limits below $4 \mathrm{mJy}$. This suggests that the fraction of cosmic star formation happening in submillimetre galaxies with $S_{850}>4$ $\mathrm{mJy}$ is less than 0.2. Even if we arbitrarily include GRB 010222 and GRB 000418 (where we have a priori knowledge of a submillimetre detection; see Table 1), this fraction is only about 0.28 . From the numbers above, we expect $0.07-0.18$ of the total cosmic star formation to have happened in submillimetre galaxies with $S_{850}>4 \mathrm{mJy}$, so this is consistent with the SCUBA results. At present the statistics from SCUBA are poor, but a moderate increase (a factor of a few) in the number of bursts observed could allow strong constraints to be placed (see Barnard et al. 2002).

There is however one potentially serious complication. All the bursts in Table 1 have detected optical afterglows. Indeed, this is perhaps the best way of determining an accurate position to allow submillimetre measurements to be made. Whether or not the optical afterglows from GRBs emerge from the host galaxies and are visible to a distant observer depends on the line-of-sight internal extinction. For UV and Class-2 SCUBA galaxies, most optical afterglows will emerge. For most submillimetre galaxies (including EROs), optical afterglows are expected to emerge only if they happen in a region of moderate obscuration. They are unlikely to emerge from well within compact molecular cores (Ramirez-Ruiz et al. 2001; Venemans \&
Blain 2001), which is the type of environment that most star formation is happening in local ULIGs (Condon et al. 1991; Surace et al. 1998; Surace \& Sanders 1999; Soifer et al. 1999).

What this means is that bursts in submillimetre galaxies may be selected against in SCUBA-observed samples since these could be the ones without optical afterglows. On the other hand, two lines of evidence suggest that this might not be a serious concern after all. First, the three bursts known to have occurred in submillimetre galaxies (GRB 010222, GRB 000418 and GRB 980703) all did have bright optical afterglows. Secondly, bursts where the optical transient has been extinguished owing to internal extinction will still have radio afterglows, and bursts with radio but no optical afterglows seem to be extremely rare: only two are known (GRB 990506 and GRB 970828).

\subsection{Radio observations of the GRB 980703 host galaxy}

The host galaxy of GRB $980703(z=0.97)$ has a high star-formation rate of about $500 \mathrm{M}_{\odot} \mathrm{yr}^{-1}$, inferred from its radio continuum luminosity (Berger et al. 2001), but only $10-30 \mathrm{M}_{\odot} \mathrm{yr}^{-1}$ inferred from optical measurements (Djorgovski et al. 1998). The reason for this disconcordance is likely to be that most of the star formation responsible for the high radio flux is happening in optically thick regions. Yet this burst had an optical afterglow, perhaps suggesting, like GRB 010222, that while most of the star formation is hidden by dust, the obscuration is not so severe that high-intensity GRB afterglows are wiped out. Again, this is not a Class-2 SCUBA galaxy, since the very bright optical afterglow (Holland et al. 2001b) did not plateau at $R \simeq 22$ at late times.

The luminosity of this host is not high enough for the $850-\mu \mathrm{m}$ flux to exceed the limit measured by Smith et al. (1999). Berger et al. (2001) estimate $S_{850}=1.9 \mathrm{mJy}$ (from the radio-far-infrared correlation. This is the only known example of a high-redshift galaxy with a submillimetre flux $S_{850}<4$ mJy with an optical counterpart and a redshift measurement.

\section{GRB HOSTS AS LOW-LUMINOSITY SUBMILLIMETRE GALAXIES}

In the previous sections we have hypothesized that much of the star formation in the Universe happened in submillimetre galaxies with $S_{850}<4 \mathrm{mJy}$, that such galaxies will be missing from both optical and submillimetre surveys, and that they may be found in samples of GRB host galaxies. They may indeed be very common in GRB host galaxy samples. If more than half the star formation in the Universe happened in such objects, then the majority of GRB host galaxies may be of this type. The fact that the host galaxy of GRB 980703, one of the first to be studied in detail at non-optical wavelengths, is such an object gives some credibility to this speculation.

We now investigate this possibility and discuss how to compile such a sample. Deep optical observations of about 30 GRB host galaxies exist, many of which would be low-luminosity submillimetre galaxies in the context of this discussion. We suggest some possible candidates. We then outline how both submillimetre and deep radio measurements of GRB hosts, like those of the GRB 980703 host (Berger et al. 2001), could be used to verify if any such candidates really are low-luminosity submillimetre galaxies. We also discuss what it could mean if submillimetre galaxies are not represented in such samples. Finally we discuss the extent of the dust obscuration in low-luminosity submillimetre galaxies and demonstrate that in the current scenario they cannot be either Class-2 SCUBA galaxies or high-redshift analogues of local ULIGs. 


\subsection{Optical measurements of host galaxies}

Optical observations are inefficient at revealing submillimetre galaxies. The reason for this is that internal extinction in submillimetre galaxies is large so that it is possible for them to have very high infrared and bolometric luminosities but low optical flux densities (Rieke \& Lebofsky 1986; Soifer et al. 1987).

Optical observations can however be used to reveal dustenshrouded star formation if the extinction is only weakly optically thick, and so the redness of a galaxy at rest-frame ultraviolet wavelengths is a measure of the amount of extinguished star formation (Meurer et al. 1995; Meurer, Heckman \& Calzetti 1999). However this is not the kind of extinction found in the more luminous submillimetre galaxies (Goldader et al. 2002). For example, the huge amount of hidden star formation in the host of GRB 980703 was not revealed by optical (rest-frame ultraviolet) measurements (Chary, Becklin \& Armus 2002). Presumably this is because this galaxy is reasonably thick, so the Meurer relation will not work. This host could well represent a typical case, meaning that optical observations of host galaxies will be of limited use in finding submillimetre galaxies. Nevertheless, a great deal of optical information about GRB host galaxies exists, and it is worth examining this data to see if we can find any clues about the nature of the galaxies. In Fig. 1 we present $R$ magnitudes and redshifts for all bursts where measurements have been made. For the 15 bursts (not including GRB 010222) with both known redshifts in the range $0.5<z<2.5$, which are likely to contribute most to total cosmic star formation and with host galaxy detections, 2 have host galaxies with $R<23$,
10 have $23.5<R<27$, and 3 have $R>27.5$. Immediately, this tells us that most of these bursts are not happening in Class-2 SCUBA galaxies, since their host galaxy $R$ magnitudes are too faint.

We studied optical images of each of the host galaxies in Table 2 and suggest that the following are the best few candidates for being submillimetre galaxies with $S_{850}<4 \mathrm{mJy}$. Our assessments are based on a number of indirect considerations e.g. similarity to the host galaxy of GRB 980703 and morphology (spiral structure, as in the case of the host of GRB 990705, suggests a host galaxy is a UV-bright galaxy as opposed to a submillimetre galaxy).

\subsubsection{GRB 000301C}

HST-STIS imaging shows this host galaxy (Fruchter \& Vreeswijk 2001 ) to be compact and only marginally resolved. The FWHM resolution of the HST image corresponds to about $1 \mathrm{kpc}$ at the redshift of this galaxy $(z=2.03)$.

\subsubsection{GRB 991208}

In HST-STIS images this host galaxy appears to be compact and only marginally resolved (Fruchter et al. 2000a), with a FWHM of only 730 pc. Furthermore, a $K$-band source (Bloom et al. 1999b) was observed coincident with this burst one week later. This could be a near-infrared afterglow, although Bloom et al. (1999b) note that this would then imply an optical-near-infrared spectral index inconsistent with the spectral index derived from optical spectroscopy. An alternative explanation is that the $K$-band light comes from an ERO component.

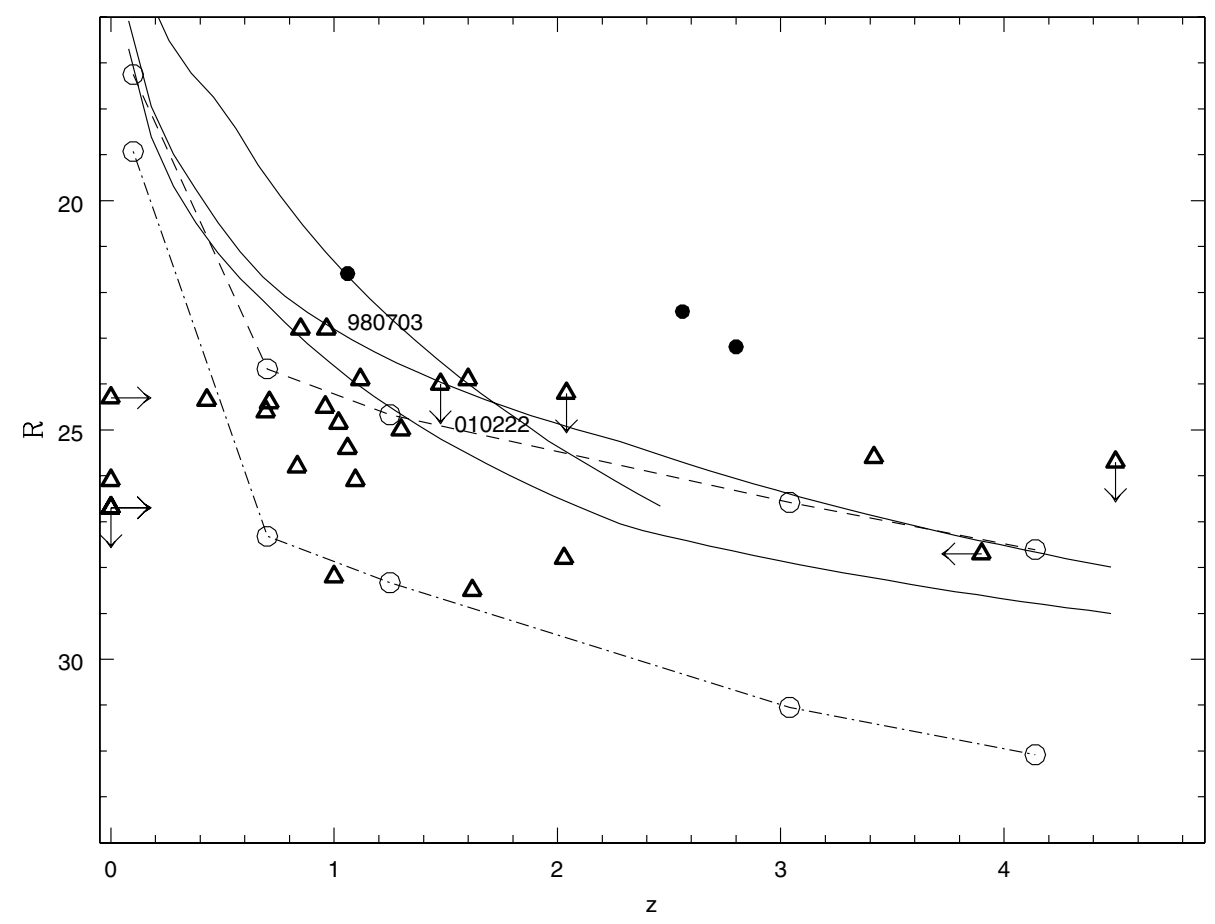

Figure 1. Optical $R$-band properties of GRB host galaxies, submillimetre galaxies and UV-bright galaxies. The triangles represent the host galaxy redshifts and magnitudes for the sample of bursts compiled in Table 2. The solid lines represent the three local ULIGs studied at ultraviolet wavelengths by Trentham et al. (1999b) as they would be seen at different redshifts. The three filled circles represent the magnitudes of Class-2 SCUBA galaxies from Smail et al. (2002) with spectroscopic redshifts, corrected for the effects of lensing. The open circles represent UV-bright galaxies of luminosity $L^{\prime}$, where $\int_{L^{\prime}}^{\infty} L \phi(L) \mathrm{d} L / \int_{0}^{\infty} L \phi(L) \mathrm{d} L=0.5$ (upper) or 0.9 (lower), at different redshifts. The luminosity function $\phi(L)$ was computed from the Las Campanas Redshift Survey (Lin et al. 1996) at $z=0$, from the survey of Cowie et al. (1999) at $z=0.7$ and $z=1.25$, and from the Lyman-break sample of Steidel et al. (1999) at $z=3$ and $z=4$. For all samples, the template Scd SED of Coleman et al. (1980) was used to convert optical luminosities to observer-frame Kron-Cousins $R$-band magnitudes. 
Table 2. Host galaxy magnitudes for GRBs, compiled and updated from Holland et al. (2000a) and Djorgovski et al. (2001a,c).

\begin{tabular}{|c|c|c|c|}
\hline Burst & Redshift (how measured) & Host $R$ mag. & Reference \\
\hline GRB 010222 & 1.48 (Mg II + Fe II abs.) & $>24$ & Djorgovski et al. (2001a) and references therein \\
\hline GRB 000926* & 2.04 (6 strong abs. lines including $\operatorname{Ly} \alpha)$ & $>24.2$ & Rol et al. (2000) \\
\hline GRB 000911* & 1.06 (host emission) & 25.4 & Lazzati et al. (2001) \\
\hline GRB 000630* & & 26.7 & Djorgovski et al. (2001a) \\
\hline GRB 000418 & $1.12(\mathrm{O}[\mathrm{II}]$ host emission) & 23.9 & Fruchter \& Metzger (2001) \\
\hline GRB $000301 C^{*}$ & 2.03 (varous weak abs. lines) & 27.8 & Fruchter \& Vreeswijk (2001) \\
\hline GRB 000131 & 4.50 (Lyman break) & $>25.7$ & Andersen et al. (2000) \\
\hline GRB $991216^{*}$ & 1.02 (Fe II $+\mathrm{Mg}_{\mathrm{I}}+\mathrm{Mg}_{\mathrm{II}}$ abs. $)$ & 24.85 & Djorgovski et al. (2001a) and references therein \\
\hline GRB 991208* & $0.71(\mathrm{O}[\mathrm{II}]+\mathrm{O}[\mathrm{III}]$ host emission $)$ & 24.4 & Djorgovski et al. (2001a) and references therein \\
\hline GRB 990712 & $0.43(\mathrm{Mg} \mathrm{II} / \mathrm{I}$ abs., $\mathrm{O}[\mathrm{II}] /[\mathrm{II}]+\mathrm{H} \gamma / \beta$ emiss.) & 24.35 & Fruchter et al. (2000b) \\
\hline GRB 990705 & 0.85 (transient X-ray abs. edge) & 22.8 & Djorgovski et al. (2001a) and references therein \\
\hline GRB 990510 & $1.62(\mathrm{Mg}$ II + Fe II abs. $)$ & 28.5 & Djorgovski et al. (2001a) and references therein \\
\hline GRB 990506 & 1.30 (see Bloom et al. 2001b) & 25.0 & Holland et al. (2000b) \\
\hline GRB 990308 & & $>26.7$ & Holland et al. (2000c) \\
\hline GRB 990123 & $1.60\left(\mathrm{Mg} I I+\mathrm{Fe}_{\mathrm{II}}\right.$ abs. $)$ & 23.9 & Djorgovski et al. (2001a) and references therein \\
\hline GRB $981226^{*}$ & & 24.30 & Holland et al. (2000d) \\
\hline GRB 980703* & 0.97 (O [II] host emission) & 22.8 & Bloom \& Kulkarni (2000) \\
\hline GRB 980613 & $1.10(\mathrm{O}[\mathrm{II}]$ host emission $)$ & 26.1 & Holland et al. (2000e) \\
\hline GRB 980519* & & 26.1 & Bloom et al. (1998b) \\
\hline GRB 980329* & $<3.9$ & 27.7 & Holland et al. (2000f) \\
\hline GRB 980326* & $\sim 1$ (putative supernova) & 28.2 & Fruchter et al. (2001a) assuming $V-R=1$ \\
\hline GRB 971214* & 3.42 (Ly $\alpha$ host emiss. + O I abs.) & 25.6 & Odewahn et al. (1998), Djorgovski et al. (2001a) \\
\hline GRB 970828 & 0.96 (host emission lines) & 24.5 & Djorgovski et al. (2001a,b) \\
\hline GRB 970508* & 0.835 (abs. + emiss. lines) & 25.8 & Castro-Tirado \& Gorosabel (1998) \\
\hline GRB 970228 & 0.70 (host emission lines) & 24.6 & Bloom et al. (2001a) \\
\hline
\end{tabular}

*Observed with SCUBA by Smith et al. (1999, 2001); see Table 1.

\subsubsection{GRB 990506}

This burst came from a compact galaxy with $r_{\mathrm{h}}=0.94 \mathrm{kpc}$. It is unusual in that it had a radio (Taylor, Frail \& Kulkarni 1999) but no optical afterglow. Possibly this is the example of a star-forming galaxy with a dense core in which the GRB occurred, with the optical afterglow missing owing to internal extinction. The optical light from the host galaxy would then trace the star formation happening at low optical depth. The $8.4-\mathrm{GHz}$ radio flux is less than $40 \mu \mathrm{Jy}$ (Taylor et al. 1999). If this is a submillimetre galaxy, it is thus likely to be a low-luminosity one unless it is at very high redshift.

\subsubsection{GRB 971214}

$H S T$ images show a compact $\left(r_{\mathrm{h}}=1.1 \mathrm{kpc}\right)$, possibly merging $V_{\mathrm{AB}} \simeq 26.5$ host galaxy (Odewahn et al. 1998). The optical colours of the burst suggest substantial internal extinction (Chary, Becklin $\&$ Armus 2002), and so it would be a good candidate for being a submillimetre galaxy. The radio imaging of this burst (Frail \& Kulkarni 1998) is moderately deep; if this is a submillimetre galaxy, its bolometric luminosity is unlikely to be more than twice that of the host galaxy of GRB 980703.

\subsubsection{GRB 970828}

Like GRB 990506, this burst had a radio, but no optical afterglow. A burst occurring in such an object would be a good candidate for having its optical (but not radio) afterglow extinguished due to dust extinction. This possibility is discussed in detail by Djorgovski et al. (2001b), whose deep ground-based optical images show that the host appears to be a merging/interacting system. They note that
HST images should soon provide important information about the morphology of the environment of this optically dark burst.

\subsubsection{GRB 970508}

HST-STIS images of this host galaxy are presented by Pian et al. (1998), but the afterglow still dominates the optical light. A subsequent image (Fruchter et al. 1998) reveals the host galaxy to be a compact, undisturbed galaxy with $r_{\mathrm{h}}=0.8 \mathrm{kpc}$.

\subsection{Compiling a sample of low-luminosity submillimetre galaxies}

An obvious question is: 'how can one compile a sample of lowluminosity submillimetre galaxies?'. This is an important exercise in that it could reveal the bulk of cosmic star formation activity taking place in the Universe.

An $850-\mu \mathrm{m}$ detection of a GRB host galaxy is the most direct method of establishing whether or not it is a submillimetre galaxy. The drawbacks of this method are that integration times to get down to the 2-mJy SCUBA confusion limit are long, and only the more luminous submillimetre galaxies, not necessarily the ones that generate most cosmic star formation, can be detected.

Radio observations of GRB host galaxies offer another powerful method. From the calibration for the Carilli-Yun radio-submm spectral index of Dunne, Clements \& Eales (2000), if we assume a radio spectral slope $\alpha=0.32$ (Berger et al. 2001) and a dust emissivity index $\beta=1.5$, then the $1.4-\mathrm{GHz}$ flux of a submillimetre galaxy at redshift $z<3$ is

$S_{1.4} \approx 900(1+z)^{-3.8}\left(\frac{S_{850}}{2 \mathrm{mJy}}\right) \mu \mathrm{Jy}$. 
The normalization of equation (1) is quite uncertain, especially at high redshifts. For example, if we use the conversion factor of Carilli \& Yun (2000), 1.4-GHz fluxes a factor of 2 lower are predicted. In our picture, many GRB host galaxies will have 1.4-GHz radio fluxes of several, maybe tens of, $\mu \mathrm{Jy}$. In other words, galaxies like the host of GRB 980703 would be common and deep radio surveys of GRB host galaxies should reveal several more.

If half of the star formation in the Universe happens in submillimetre sources with $S_{850}$ between 0.4 and $4 \mathrm{mJy}$, at redshifts of about 2, then we would expect about half of GRB host galaxies to have $1.4-\mathrm{GHz}$ fluxes $S_{1.4}>3 \mu \mathrm{Jy}$. If we restrict a survey to GRB hosts with known redshifts $z<1$, then we would expect about half of GRB host galaxies to have $S_{1.4}>13 \mu \mathrm{Jy}$. For comparison, the host galaxy of GRB 980703 has $S_{1.4}=68 \mu$ Jy (Berger et al. 2001), the high flux reflecting the relatively low redshift $z=0.97$ of this burst. At the VLA, ${ }^{1}$ a $1 \sigma$ sensitivity $S_{1.4} \simeq 5 \mu \mathrm{Jy}$ can be achieved in $24 \mathrm{~h}$. From equation (1), $S_{1.4}$ is a steeply decreasing function of redshift. This suggests that low-redshift GRBs like GRB 990712 ( $z=0.43$ ) would be the best targets for deep radio imaging.

Imaging of GRB host galaxies at a wavelength $70 \mu \mathrm{m}$ with $S I R T F^{2}$ may help too, particularly for the lower-redshift objects. Direct submillimetre surveys with ALMA will uncover such objects, and we will no longer need to rely on using GRBs as beacons. ALMA will also be able to detect a 1-mJy host galaxy in less than a minute, and so can be used to image all known GRB positions in a matter of a few weeks of observations.

Should submillimetre host galaxies not turn up in any of the samples described in this section, this would also be an interesting result. One possibility could be that GRBs avoid submillimetre galaxies because the environments there are not conducive to the physical processes responsible for generating GRBs (metallicity, likely to be high in submillimetre-luminous galaxies, could play a role for example; see Ramirez-Ruiz, Lazzati \& Blain 2002). Alternatively it could mean that our estimates of $f_{\text {submm }}$ are too high, possibly because the assumptions made in modelling the luminosity evolution of submillimetre galaxies are flawed, for example if high-redshift submillimetre galaxies actually have dust temperatures systematically less than the $40 \mathrm{~K}$ that seems to be typical.

\subsection{Obscuration in low-luminosity submillimetre GRB host galaxies}

Host galaxies like that of GRB 980703 appear to have star formation that is only lightly obscured, intermediate between Class-2 SCUBA galaxies (which would have brighter $R$ magnitudes) and ULIG cores (which would extinguish optical afterglows).

The occasions where GRBs did appear to happen in dense cores were the two occasions (GRB 970828 and GRB 990506) where radio but not optical afterglows were detected. That this has been observed twice suggests that star formation in dense cores at high redshifts is not as rare as at low redshifts, where the contribution of ULIGs to the star formation rate at $z=0$ (Gallego et al. 1995) is negligible.

However, the majority of high-redshift submillimetre galaxies, if the hosts of GRB 010222, GRB 000418 and GRB 980703 are at all representative, are probably not of this type. Their gas and dust are likely to be less concentrated. Such a scenario is required if most of the stars currently seen in local galaxies formed in submillimetre

\footnotetext{
${ }^{1}$ http: //www.aoc.nrao.edu/vla/html/VLAhome.shtml

${ }^{2}$ http://sirtf.caltech.edu/
}

galaxies (Trentham 2001). These submillimetre galaxies would be intermediate in terms of structural parameters between Lyman-break galaxies and ULIGs, possibly of the type hypothesized to exist on the boundary of detectability in both the optical and submillimetre wavebands by Adelberger \& Steidel (2000).

\section{ACKNOWLEDGMENTS}

We thank Vicki Barnard, Fiona Harrison, Davide Lazzati, Priya Natarajan, Martin Rees, Dave Sanders, Nial Tanvir and Bram Venemans for helpful conversations, and the anonymous referee for useful comments on the manuscript. We thank Jeff Goldader for making the data and results from the HST-STIS imaging program of local infrared-luminous galaxies available and Ian Smail for providing the SCUBA source catalogue paper prior to publication. ERR acknowledges support from CONACYT, SEP and the ORS foundation. AWB acknowledges the Raymond \& Beverly Sackler Foundation for financial support at the IoA. This work has used HST data provided by the Survey of the Host Galaxies of Gamma-Ray Bursts (http://www.ifa.au.dk/ hst/grb-hosts/data/ index.html).

\section{REFERENCES}

Adelberger K. L., Steidel C. C., 2000, ApJ, 544, 218

Amati L. et al., 2000, Sci, 290, 953

Andersen M. I. et al., 2000, A\&A, 364, L54

Barnard V. E. et al., 2002, MNRAS, submitted

Berger E., Kulkarni S. R., Frail D. A., 2001, ApJ, 560, 652

Berger E., Cowie L., Aussel H., Barger A., Kulkarni S. R., Frail D., 2002, GRB Coordinates Network (GCN), 1182

Björnsson G., Hjorth J., Jakobsson P., Christensen L., Holland S., 2001, ApJ, 552, L121

Blain A. W., 2001, in Lutz D., Tacconi L., eds, Starbursts Near and Far. Springer-Verlag, Heidelberg, p. 303

Blain A. W., Ivison R. J., Smail I., 1998, MNRAS, 296, L29

Blain A. W., Smail I., Ivison R. J., Kneib J.-P., 1999a, MNRAS, 302, 632

Blain A. W., Jameson A., Smail I., Longair M. S., Kneib J.-P., Ivison R. J., 1999b, MNRAS, 309, 715

Blain A. W., Smail I., Ivison R. J., Kneib J.-P., Frayer D. T., 2002, Phys. Rep., in press (astro-ph/0202228)

Bloom J. S., Kulkarni S. R., 2000, GCN, 702

Bloom J. S., Djorgovski S. G., Kulkarni S. R., Frail D. A., 1998a, ApJ, 507, L25

Bloom J. S., Kulkarni S. R., Djorgovski S. G., Gal R. R., Eichelberger A., Frail D. A., 1998b, GCN, 149

Bloom J. S. et al., 1999a, Nat, 401, 453

Bloom J. S., Diercks A., Kulkarni S. R., Djorgovski S. G., Scoville N. Z., Frayer D. T., 1999b, GCN, 480

Bloom J. S., Djorgovski S. G., Kulkarni S. R., 2001a, ApJ, 554, 678

Bloom J. S., Frail D. A., Sari R., 2001b, AJ, 121, 2879

Carilli C. L., Yun M. S., 1999, ApJ, 513, L13

Carilli C. L., Yun M. S., 2000, ApJ, 530, 618

Castro-Tirado A. J., Gorosabel J., 1998, IAU Circ. 6848

Chary R., Elbaz D., 2001, ApJ, 556, 562

Chary R., Becklin E. E., Armus L., 2002, ApJ, 566, 229

Coleman G. D., Wu C.-C., Weedman D. W., 1980, ApJS, 43, 393

Condon J. J., Huang Z.-P., Yin Q. F., Thuan T. X., 1991, ApJ, 378, 65

Cowie L. L., Songaila A., Barger A. J., 1999, AJ, 118, 603

Djorgovski S. G., Kulkarni S. R., Bloom J. S., Goodrich R., Frail D. A., Piro L., Palazzi E., 1998, ApJ, 508, L17

Djorgovski S. G. et al., 2001a, in Gurzadyan V., Jantzen R., Ruffini R., eds, Proc. IX Marcel Grossmann Meeting. World Scientific, Singapore, in press (astro-ph/0106574)

Djorgovski S. G., Frail D. A., Kulkarni S. R., Bloom J. S., Odewahn S. C., Diercks A., 2001b, ApJ, 562, 654 
Djorgovski S. G. et al., 2001c, in Masetti N. et al., eds, Gamma-Ray Bursts in the Afterglow Era: 2nd Workshop. Springer-Verlag, Berlin, in press (astro-ph/0107535)

Draine B. T., Hao L., 2002, ApJ, 569, 780

Dunne L., Clements D. L., Eales S. A., 2000, MNRAS, 319, 813

Eales S., Lilly S., Gear W., Dunne L., Bond J. R., Hammer F., Le Févre O., Crampton D., 1999, ApJ, 515, 518

Eales S., Lilly S., Webb T., Dunne L., Gear W., Clements D., Yun M., 2000, AJ, 120, 2244

Frail D., Kulkarni S. R., 1998, GCN, 3

Frail D. et al., 2002, ApJ, 565, 829

Fruchter A. S., Metzger M. R., 2001, GCN, 1061

Fruchter A. S., Vreeswijk P., 2001, GCN, 1063

Fruchter A. et al., 1998, http: //www-int.stsci.edu/ fruchter/GRB/ 970508/august-1998.html

Fruchter A. S., Vreeswijk P., Sokolov V., Casto-Tirado A., 2000a, GCN, 872

Fruchter A., Sahu K., Gibbons R., Petro L., Ferguson H., 2000b, GCN, 565

Fruchter A. S., Vreeswijk P., Nugent P., 2001a, GCN, 1029

Fruchter A. S., Krolik J. H., Rhoads J. E., 2001b, ApJ, 563, 597

Galama T. J., Wijers R. A. M. J., 2001, ApJ, 549, L209

Gallego J., Zamorano J., Aragón-Salamanca A., Rego M., 1995, ApJ, 455, L1

Gispert R., Lagache G., Puget J.-L., 2000, A\&A, 360, 1

Goldader J. D., Meurer G. R., Heckman T. M., Seibert M., Sanders D. B., Calzetti D., Steidel C. C., 2002, ApJ, 568, 651

Holland W. S. et al., 1999, MNRAS, 303, 659

Holland S. et al., 2000a, GCN, 698

Holland S. et al., 2000b, GCN, 731

Holland S. et al., 2000c, GCN, 726

Holland S. et al., 2000d, GCN, 749

Holland S. et al., 2000e, GCN, 777

Holland S. et al., 2000f, GCN, 778

Holland S., Fynbo J., Gorosabel J., Henden A., Hjorth J., Jensen B., Pedersen H., 2001a, GCN, 1002

Holland S. et al., 2001b, A\&A, 371, 52

Ivison R., Smail I., Le Borgne J.-F., Blain A. W., Kneib J.-P., Bezecourt J., Kerr T. H., Davies J. K., 1998, MNRAS, 298, 583

Ivison R., Smail I., Barger A. J., Kneib J.-P., Blain A. W., Owen F. N., Kerr T. H., Cowie L. L., 2000, MNRAS, 315, 209

Ivison R. J., Smail I., Frayer D. T., Kneib J.-P., Blain A. W., 2001, ApJ, 561, L45

Katz J., 1994, ApJ, 422, 248

Kulkarni S. R. et al., 1998, Nat, 395, 663

Lazzati D. et al., 2001, A\&A, 378, 996

Lazzati D., Covino S., Ghisellini G., 2002, MNRAS, 330, 583

Lin H., Kirshner R. P., Shectman S. A., Landy S. D., Oemler A., Tucker D. L., Schechter P. L., 1996, ApJ, 464, 60

Lonsdale C., 2001, BAAS, 198, 2502
MacFadyen A. I., Woosley S. E., 1999, ApJ, 524, 262

MacFadyen A., Woosley S. E., Heger A., 2001, ApJ, 550, 410

Meurer G. R., Heckman T. M., Leitherer C., Kinney A., Robert C., Garnett D. R., 1995, AJ, 110, 2665

Meurer G. R., Heckman T. M., Calzetti D., 1999, ApJ, 521, 64

Mészáros P., Rees M. J., 1997, ApJ, 476, 232

Odewahn S. C. et al., 1998, ApJ, 509, L5

Paczyński B., 1998, ApJ, 494, L45

Paczyński B., Xu G., 1994, ApJ, 427, 708

Peacock J. A. et al., 2000, 318, 535

Pettini M., Shapley A. E., Steidel C. C., Cuby J.-G., Dickinson M., Moorwood A. F. M., Adelberger K. L., Giavalisco M., 2001, ApJ, 554, 981

Pian E. et al., 1998, ApJ, 492, L103

Piro L. et al., 2000, Sci, 290, 955

Ramirez-Ruiz E., Trentham N., Blain A. W., 2001, MNRAS, 329, 465

Ramirez-Ruiz E., Lazzati D., Blain A. W., 2002, ApJ, 565, L9

Rees M. J., Mészáros P., 1992, MNRAS, 258, 41

Rees M. J., Mészáros P., 1994, ApJ, 430, L93

Reichart D. E., 1999, ApJ, 521, L111

Reichart D. E., Price P. A., 2002, ApJ, 565, 174

Rieke G. H., Lebofsky M. J., 1986, ApJ, 304, 326

Rol E., Vreeswijk P. M., Tanvir N., 2000, GCN 850

Sanders D. B., Mirabel I. F., 1996, ARA\&A, 34, 749

Sari R., Piran T., 1995, ApJ, 455, L143

Scoville N. Z. et al., 1998, ApJ, 492, L107

Smail I., Ivison R. J., Blain A. W., Kneib J.-P., 2002, MNRAS, 331, 495

Smith I. A. et al., 1999, A\&A, 347, 92

Smith I. A., Tilanus R. P. J., Wijers R. A. M. J., Tanvir N., Vreeswijk P., Rol E., Kouveliotou C., 2001, A\&A, 380, 81

Soifer B. T., Sanders D. B., Madore B. F., Neugebauer G., Danielson G. E., Elias J. H., Lonsdale C. J., Rice W. L., 1987, ApJ, 320, 238

Soifer B. T., Neugebauer G., Matthews K., Becklin E. E., Ressler M., Werner M. W., Weinberger A. J., Egami E., 1999, ApJ, 513, 207

Steidel C. C., Adelberger K. L., Giavalisco M., Dickinson M., Pettini M., 1999, ApJ, 519, 1

Surace J. A., Sanders D. B., 1999, ApJ, 512, 162

Surace J. A., Sanders D. B., Vacca W. D., Veilleux S., Mazzarella J. M., 1998, ApJ, 492, 116

Taylor G., Frail D. A., Kulkarni S. R., 1999, GCN, 350

Trentham N., 2001, MNRAS, 323, 542

Trentham N., Blain A. W., Goldader J., 1999a, MNRAS, 305, 61

Trentham N., Kormendy J., Sanders D. B., 1999b, AJ, 117, 2152

Venemans B. P., Blain A. W., 2001, MNRAS, 325, L1777

Waxman E., Draine B. T., 2000, ApJ, 537, 796

Woosley S. E., 1993, ApJ, 405, 273

This paper has been typeset from a $\mathrm{T}_{\mathrm{E}} \mathrm{X} / \mathrm{L} \mathrm{A} \mathrm{E} \mathrm{X}$ file prepared by the author. 